\title{
Efficient Methodology for 3D Statistical Reconstruction of High Resolution Coplanar PET/CT Scanner
}

Juan E. Ortuño, José L. Rubio, Student Member, IEEE, Pedro Guerra, Member, IEEE, George Kontaxakis, Senior Member, IEEE, Manuel Desco, Juan J. Vaquero, Senior Member, IEEE, and Andrés Santos, Senior Member, IEEE

\begin{abstract}
A fully 3D statistical image reconstruction algorithm has been developed for a high-resolution coplanar PET/CT scanner based on rotating planar PET detectors. The system matrix has been modeled with custom Monte Carlo techniques optimized for the specific scanner architecture. The system model includes positron range, non-colinearity of gamma rays and crystal interaction modelling with attenuation and Compton scattering effects. Only $0.21 \%$ of the system matrix columns are modeled in detail, obtaining the rest of the values with axial and transaxial voxel-driven symmetries. The iterative algorithm is a fully 3D approach, regularized with the anatomical registered image using a novel version of the minimum cross entropy (MXE) scheme, and accelerated employing ordered subsets. The proposed method has been shown to produce images with superior quality than 3D hybrid (FORE+2D-OSEM) algorithms applied on synthetic GATE data, as well as on real small animal acquisitions.
\end{abstract}

\section{INTRODUCTION}

$\mathbf{P}^{\text {REClinical PET scanners are designed to achieve high }}$ Ppatial resolution to detect small animal functional target volumes. One affordable solution consists of opposed planar detectors mounted on a rotating gantry. This configuration can achieve state-of-the-art spatial resolution at the cost of lower detection sensitivity than full ring alternatives.

The rotational PET (rPET) [1] (SUINSA Medical Systems, Spain) pre-clinical camera consists of two pairs of planar detector blocks, composed of pixelated scintillator crystals and PS-PMTs. The gantry has $370^{\circ}$ rotation span. This design has been recently upgraded to incorporate multimodality capabilities by integrating an X-ray tube and a semiconductor $\mathrm{X}$-ray detector [2]. The PET block detectors are disposed in "V" arrangements as seen in Fig. 1 to allow enough space to accommodate the X-ray CT system with cone beam geometry

Manuscript received November 14, 2008. This work has been partly funded by the CDTEAM project and CENIT programme (Spanish Ministry of Industry), EMIL (EU Network of Excellence), CIBER CB07/09/0031 and RETIC-RECAVA (Spanish Ministry of Health) and TEC2007-64731/TCM (Spanish Ministry of Education and Science).

J. E. Ortuño, J. R Rubio, G. Kontaxakis and A. Santos are with the Biomedical Image Technology Group, Dpto. Ingenieria Electrónica, Universidad Politécnica de Madrid, Spain, and with the Biomedical Research Center on Bioengineering, Biomaterials and Nanomedicine (CIBER-BBN), Madrid, Spain E-28040 (e-mail: juanen, jlrubio, gkont, andres@die.upm.es).

P. Guerra is with the Biomedical Research Center of Bioengineering, Biomaterials and Nanomedicine (CIBER-BBN), Madrid, Spain E-28040 (email: pguerra@ciber-bbn.es).

M. Desco and J. J. Vaquero are with the Unidad de Medicina y Cirugia Experimental. Hospital General Universitario Gregorio Marañón, Madrid, Spain E-28007 (e-mail: desco, juanjo@mce.hggm.es). in-plane and on the same rotational gantry. Opposite detectors are separated by $140 \mathrm{~mm}$. Each detector is composed of a $30 \times 30$ array of $1.5 \times 1.5 \times 12 \mathrm{~mm}$ LYSO crystals, coupled to a flat panel-type large area PS-PMTs.

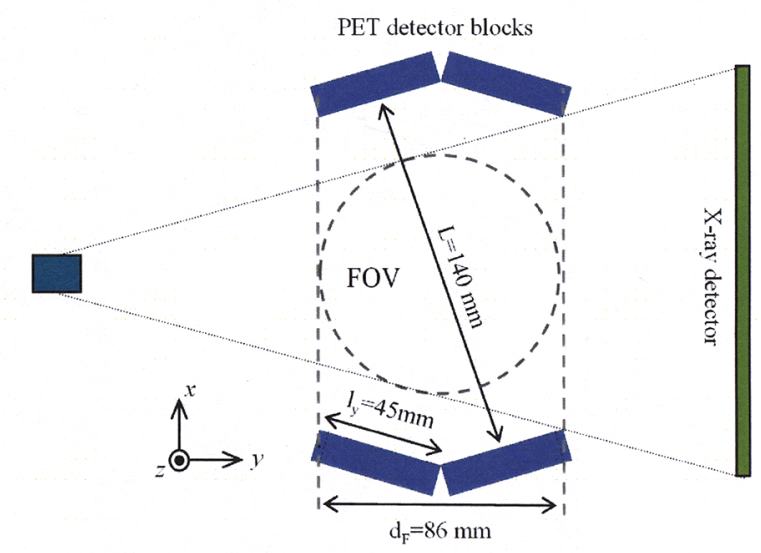

Fig. 1. VrPET/CT transaxial view. A X-ray flat panel detector and four gamma ray detector blocks are mounted on a common gantry with $370^{\circ}$ rotation span. Coincidences are allowed between four pairs of detector blocks.

The VrPET/CT scanner has higher sensitivity for PET in the center of the FOV than the previous rPET design, since four pairs of detectors are now enabled in coincidence mode, instead of two of the former camera. Furthermore, the FOV diameter within complete angular projections reaches $86 \mathrm{~mm}$ in the transaxial plane.

We have developed an efficient methodology for 3D statistical reconstruction for this system. The proposed iterative algorithms improve the computational efficiency and the image quality of methods previously applied to rPET [3] but adapted to the new geometry, also being able to use CT co-registered anatomical information.

\section{MATERIALS AND METHODS}

The OSEM (ordered subsets-expectation maximization) algorithm [4] with pre-calculated system matrix modeled with Monte Carlo methods and stored in sparse format, has been demonstrated to be a powerful and computationally efficient 
reconstruction technique applied to high-resolution rotational PET scanners [3].

The designed and developed algorithm for multimodal PET/CT acquisitions is a $3 \mathrm{D}$ generalization of an ordered subsets (OS) version of the MXE (minimum cross-entropy) algorithm $[5,6]$ :

$$
\mathbf{x}^{(n+1)}=\mathbf{x}^{(n)} \frac{1}{\mathbf{A}^{\mathbf{T}} \mathbf{I}}\left[\mathbf{A}^{\mathbf{T}} \frac{\mathbf{y}}{\mathbf{A} \mathbf{x}^{(n)}}-\beta \ln \left(\frac{\mathbf{x}^{(n)}}{f\left(\mathbf{x}^{(n-1)}, \mathbf{z}\right)}\right)\right]
$$

where $\mathbf{x}$ is the vector of image basis functions, $\mathbf{y}$ is the vector of projection elements, and $\mathbf{A}$ is the system matrix (SM). The cross-entropy term regularizes the characteristic noise amplification properties of OSEM algorithm. The function $f(\cdot)$ contained in the denominator is a gradient inverse weighted smoothing [7] extended here to 3D kernels. This gradient is a function of the result of the previous iteration and the anatomical priors $\mathbf{z}$, i.e, the CT registered scan. The global weighting parameter $\beta$ can be empirically adjusted to obtain a desired noise-resolution ratio and guarantee the positivity constraints.

The VrPET/CT camera histograms list-mode data into $30^{2}$ direct and oblique sinograms with 117 radial and 190 angular bins each one. 3D FOV is discretized in an orthogonal grid with voxels of $0.5 \times 0.5 \mathrm{~mm}^{2}$ in the transaxial plane and $0.375 \mathrm{~mm}$ in the axial direction. Small animal studies require a $60 \mathrm{~mm}$ transaxial FOV diameter, with 11288 voxels contained in each transaxial plane: $\left(120^{2}(\pi / 4) \approx 11288\right)$.

The high number of SM elements (see Table I for two different voxel sizes in $z$ ) imposes important computing demands in terms of time required to complete the iterative reconstruction procedures and the computer memory needed for storage of the pre-calculated SM.

TABLE I

SIZE AND NUMBER OF ELEMENTS OF THE PRECALCULATED SM

\begin{tabular}{lll}
\hline Number of Voxels & $120 \times 120 \times 60$ & $120 \times 120 \times 120$ \\
Voxel size $(\mathrm{mm})$ & $0.5 \times 0.5 \times 0.75$ & $0.5 \times 0.5 \times 0.375$ \\
SM elements & $9.8 \times 10^{12}$ & $1.9 \times 10^{13}$ \\
Modelled voxels & 1432 & 2864 \\
Precalculated SM size & $1.43 \mathrm{~GB}$ & $2.83 \mathrm{~GB}$ \\
\hline
\end{tabular}

Rotational $\left(90^{\circ}, 180^{\circ}\right.$ and $\left.270^{\circ}\right)$ and reflection symmetries in the transaxial plane [8] are calculated in a voxel-driven scheme, computing approximately $1 / 8^{\text {th }}$ of the voxels contained in a single slice (Fig. 2).

Rotational symmetry requires an angular shift and eventually a radial inversion of the associated sinogram bin, and can be computed within each subset projection and backprojection step. Reflection symmetry involves an angular inversion that connects sinogram positions belonging to different subsets. In the iterative image reconstruction process, the pre-calculated SM values are loaded in an efficient order to optimize RAM performance associated to the selection of subsets.

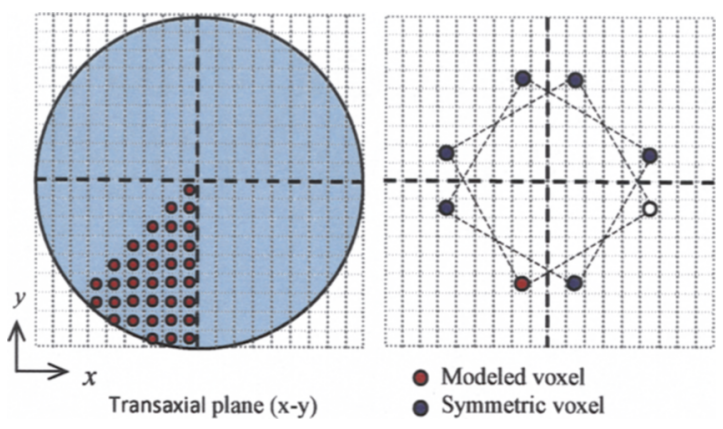

Fig. 2. Schematic model of voxels located in the transaxial plane. Only the SM columns involving the $1 / 8$ th of the voxels inscribed in the FOV circle need to be modeled and stored. The rest of SM values can be obtained by applying rotations and reflections.

Exact rotational symmetries without approximate interpolations require even numbers of radial bins in each subset. The total 190 discrete samples in the VrPET/CT scanner allow the use of 19 or 5 subsets (with 10 or 38 angular discrete angular projections in each subset).

Translation symmetry is applied choosing the voxel size along the z-axis being divisor of the pixelated crystal row size. Reflection axial symmetry also needs proper alignment between the orthogonal grid and the pixilated crystals.

The optimum axial alignment requires two central slices to be modeled in detail for voxel size of $0.375 \mathrm{~mm}$ in z. For coarser grid $(0.75 \mathrm{~mm})$ the modeling overhead is reduced to only one slice. When both axial and transaxial symmetries are employed, $0.21 \%$ of the SM columns need to be modeled in detail, that is 2864 out of 1354560 voxels using the finest grid (Table I).

The image and projection vectors are reorganized to optimize axial translation operations, which are the most time consuming symmetry-related tasks. In the projection and back-projection steps, the axial symmetry loop is nested into the transaxial symmetry loop. A voxel-level parallelization in a shared memory scheme has been employed, allowing 4 simultaneous threads for each step in an Intel Core $^{\mathrm{TM}} 2$ Quad processor. Reconstruction times have been reduced from 12 min to 3 min per complete iteration over all subsets (i.e, 19 sub-iterations). For coarser grid $(0.75 \mathrm{~mm})$ in the axial axis, the computing time per iteration is reduced to only $1 \mathrm{~min} 27 \mathrm{~s}$.

Pre-calculated non-zero SM values are stored in disk, sorted by subset in a sparse format way. The statistical image quality depends strongly on the accuracy of the model. Monte Carlo custom methodologies [3] developed for the rPET camera have been adapted and optimized for the new VrPET geometry.

The methods developed model positron range and noncolinearity of gamma ray emissions, which depend on the radioisotope and the material of which the object imaged is composed. Biological tissue has been approximated $a$ priori as water. In small animal studies with F-18 tracers, the detector response becomes a critical factor for accurate SM modelling. 
Gamma penetration and Compton scattering in scintillation crystals is carefully simulated with attenuation cross-sections tables and the Klein-Nishina formula. To avoid poor sparseness of the SM with negligible values, an auxiliary LUT is employed, which contains the relative probabilities of detection in neighboring crystals, according to the angle and point of intersection of the gamma rays. The pre-calculated $\mathrm{SM}$ values are then stored in less than $3 \mathrm{~GB}$ for the configurations described above

\section{RESULTS}

\section{A. Derenzo-Type phantoms}

The proposed image reconstruction scheme has been evaluated with data generated using the GATE platform [9]. A Derenzo-type phantom was simulated with water-filled rods of $\{4.8,3.6,2.4,1.8,1.2\} \mathrm{mm}$ diameter and $16 \mathrm{~mm}$ length. Activity density was selected to be $5 \mu \mathrm{Ci} / \mathrm{cm}^{3} \quad(\mathrm{~F}-18$ radiopharmaceutical). $\mathrm{A}$ total amount of 10.8 million coincidences was collected with an energy window of 400-700 $\mathrm{keV}$ and 12 ns coincidence window.

The phantom was situated with rods perpendicular to the scanner axial axis, in order to better evaluate the spatial resolution in the axial direction. No anatomical information was used in this experiment.

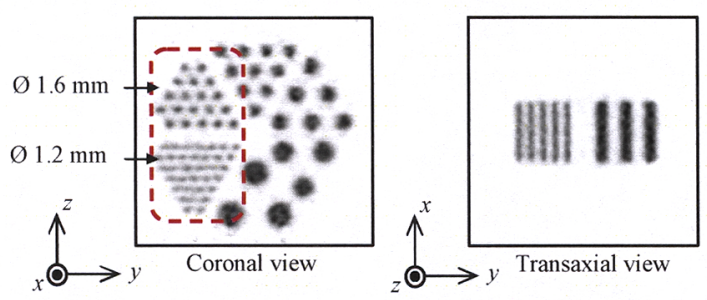

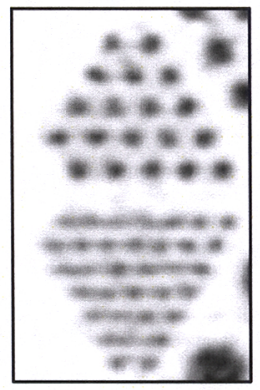

(a)

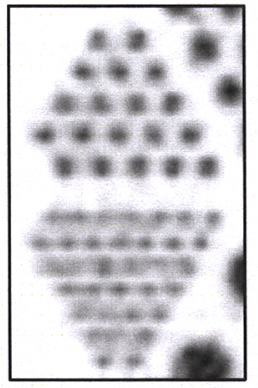

(b)

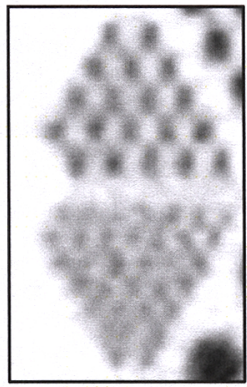

(c)
Fig. 3. GATE acquisition of Derenzo-type phantom with rods aligned with the transaxial plane: Partial coronal views. (a) 3D OS-MXE reconstruction with pixel size of $0.5 \times 0.5 \times 0.375 \mathrm{~mm}$. (b) $3 \mathrm{D}$ OS-MXE reconstruction with pixel size of (b) $0.5 \times 0.5 \times 0.75 \mathrm{~mm}$. (c) FORE \& 2 DOSEM reconstruction.

Fig. 3 shows a partial view of the Derenzo-type phantom acquisition. Rods have better contrast in $3 \mathrm{D}$ mode with voxel size of $0.375 \mathrm{~mm}$ axially. 2D-OSEM with Fourier rebinning (FORE) shows poorer results in terms of contrast between rods. The voxel size in this case is $0.75 \mathrm{~mm}$ axially. 5 iterations with 19 subsets per iteration were employed in all cases.

Fig. 4 shows a surface rendering of the partial views shown in Fig. 3, for better appreciating the better contrast of 3DMXE reconstruction using the finest grid.

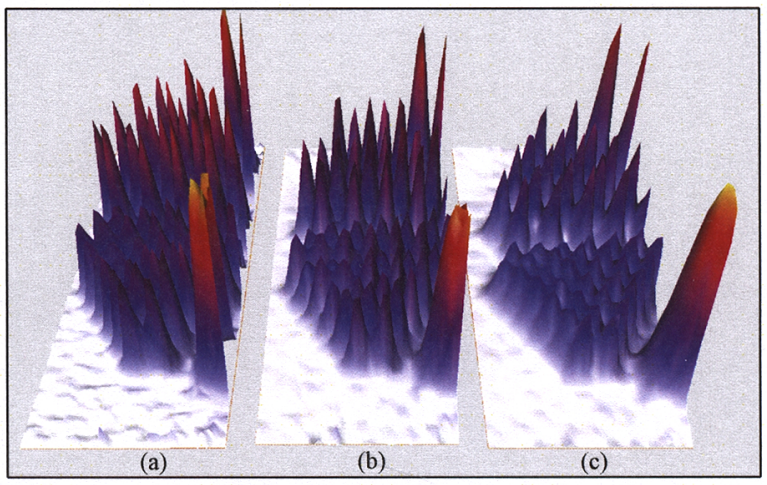

Fig. 4. Surface rendering of gray level images represented in Fig. 3. (a) 3D OS-MXE reconstruction with pixel size of $0.5 \times 0.5 \times 0.375 \mathrm{~mm}$. (b) $3 \mathrm{D}$ OSMXE reconstruction with pixel size of (b) $0.5 \times 0.5 \times 0.75 \mathrm{~mm}$. (c) FORE \& $2 \mathrm{D}$ OSEM reconstruction.

\section{B. Phantom with registered anatomical information}

A quality phantom has been modeled with GATE in order to evaluate the noise characteristics and edge contrast in presence of registered anatomical data.

This phantom consists of several rods filled with water containing F-18, embedded in a main chamber cylinder (or background) with $1 / 5^{\text {th }}$ of activity density. $5.2 \times 10^{6}$ coincidences were collected with the same energy and coincidence window of the Derenzo-type experiment. A scheme of the activity distribution is shown in Fig. 5 (up) with eight hot rods of $\{8,7,6,5,4,3,2,1\} \mathrm{mm}$ diameter and 25 $\mathrm{mm}$ length in the upper part (as seen in Fig. 5), and 2 hot cylinders of $16 \mathrm{~mm}$ diameter and $9 \mathrm{~mm}$ length in the lower part of the phantom. There are anatomical boundaries without activity gradient for evaluating the robustness of the reconstruction algorithm against anatomical information not correlated with functional PET regions (see the anatomical map of Fig. 5)

In Fig. 6, 3D OS-MXE reconstructions of the phantom study are shown at three transaxial and one coronal view. The finest grid was used (i.e, $0.5 \times 0.5 \times 0.375 \mathrm{~mm}$ ) with 4 iterations and 19 subsets per iteration (i.e, 76 sub-iterations). The edges aligned with anatomical interfaces are enhanced, while there are no significant artifacts related to non-correlated anatomical (i.e, simulated CT) and PET data. 


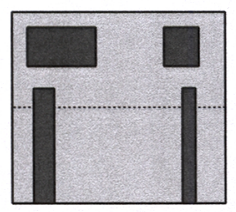

Transaxial

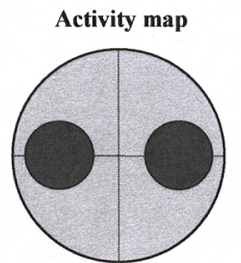

Coronal (upper cyl.)

Anatomical (CT) map

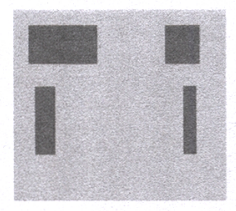

Transaxial

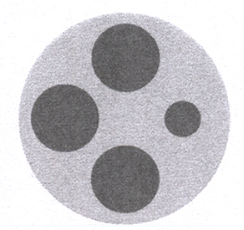

Coronal (upper cyl.)

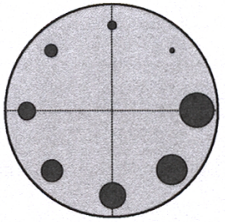

Coronal (lower rods)

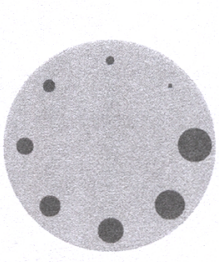

Coronal (lower rods)
Fig. 5. Schematical representation of the of quality phantom simulated with GATE. Hot rods filled with water labeled with F-18 are aligned with anatomical boundaries

Table 2 shows the coefficient of variation $(\mathrm{CoV})$ in regions of interest containing active rods of different radii (see Fig. 5) as well as the background, compared with results obtained without anatomical data using 3D-OSEM reconstruction regularized with MRP scheme.

\section{TABLE 2}

COEFFICIENT OF VARIATION (COV) OF REGIONS OF INTEREST WITH OSEM AND MXE RECONSTRUCTION METHODS

\begin{tabular}{lll}
\hline Region of intererest & 3D-MXE & 3D-OSEM \\
$\emptyset 5 \mathrm{~mm}$ rod & 0.44 & 0.47 \\
$\emptyset 6 \mathrm{~mm}$ rod & 0.72 & 1.00 \\
$\emptyset 7 \mathrm{~mm}$ rod & 0.96 & 1.18 \\
$\emptyset 8 \mathrm{~mm}$ rod & 0.93 & 1.15 \\
background & 0.14 & 0.20 \\
\hline
\end{tabular}

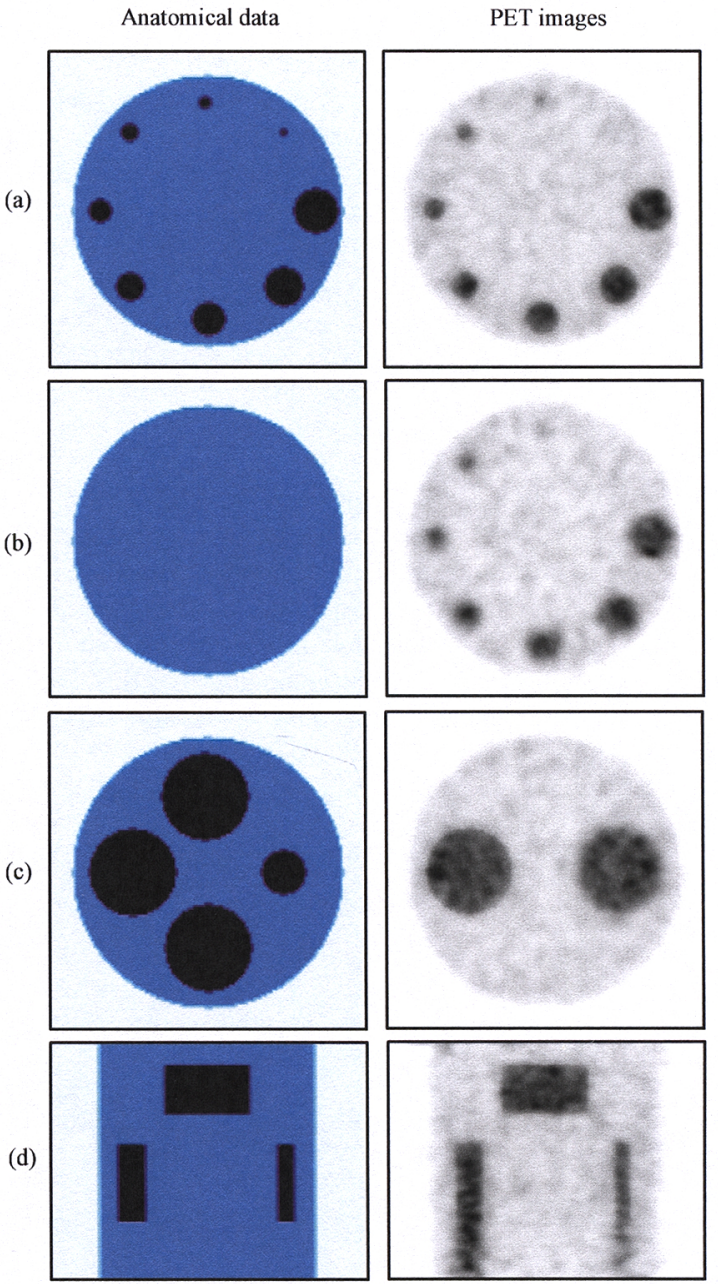

Fig. 6. OS-MXE reconstructions of the synthetic phantom with registered anatomical images (blue palette pictures). (a) Transaxial view of region with small rods with aligned anatomical information. (b) Transaxial view where the small diameter rods were not modeled with anatomical gradients, showing poorer edge resolution. (3) Transaxial view of upper part of the phantom, showing enhanced edge resolution in the cylinder with aligned anatomical data. (d) Coronal view of the phantom

\section{Rat study}

Experimental acquisition with rat injected with F-18-FDG revealed superior spatial resolution for the $3 \mathrm{D}$ method in the axial direction. Fig. 7 shows that contrast between functional structures located in the vertebral column of a rat study is superior in the $3 \mathrm{D}$ reconstructed image. 


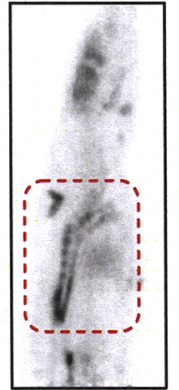

(a)

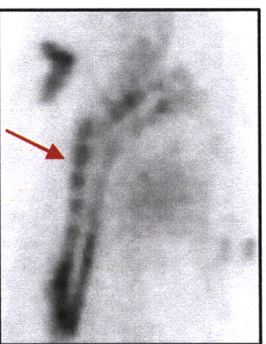

(b)

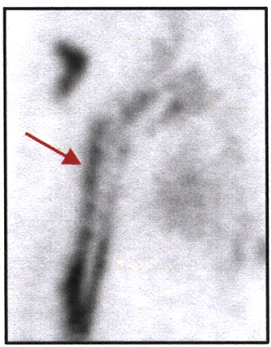

(c)
Fig. 7. (a) Coronal view of rat injected with FDG and reconstructed with 3D OS-MXE algorithm; (b) Detail showing vertebrae; (c) FORE \& 2D-OSEM reconstruction.

\section{CONCLUSSIONS}

We have proposed a novel fully $3 \mathrm{D}$ algorithm for the iterative reconstruction of PET data, taking into consideration anatomical correlated data, based on the maximum entropy approach and accelerated using the ordered subsets technique. The algorithm has been validated on the $\mathrm{VrPET} / \mathrm{CT}$ rotational camera. The anatomical correlates correspond to the CT images obtained with this system.

For simulation data using the GATE platform the proposed 3D OS-MXE method demonstrates superior image quality over the FORE-2D-OSEM alternative in terms of spatial resolution in the axial axis. The edges aligned with anatomical interfaces are enhanced while there are no significant artifacts related to non-correlated anatomical data.

In real data acquisitions with small animals, even without the anatomically correlated images, a substantial improvement is obtained in the axial spatial resolution over FORE-2DOSEM method. Future work will include CT registered data to evaluate edge improvements with rat studies.

\section{REFERENCES}

[1] J. J. Vaquero, E. Lage, L. Ricón, M. Abella, E. Vicente, and M. Desco, "rPET detectors design and data processing", at Nuclear Science Symposium Conference Record, IEEE, vol 5, pp. 2885-2889, Puerto Rico (USA) 2005.

[2] J. J. Vaquero, et al., "Co-planar PET/CT for small animal imaging", at Nuclear Science Symposium Conference Record, IEEE, vol 3, pp. 17481751, Puerto Rico (USA) 2005.

[3] J. E. Ortuño, P. Guerra, J. L. Rubio, G. Kontaxakis, and A. Santos, "3DOSEM iterative image reconstruction for high-resolution PET using precalculated system matrix", Nuclear Instruments \& Methods in Physics Research Section a-Accelerators Spectrometers Detectors and Associated Equipment, vol. 569, no. 2, pp. 440-444, 2006.

[4] H. M. Hudson and R. S. Larkin, "Accelerated image-reconstruction using ordered subsets of projection data", IEEE Transactions on Medical Imaging, vol. 13, no. 4, pp. 601-609, 1994.

[5] B. A. Ardekani, M. Braun, B. F. Hutton, I. Kanno, and H. lida, "Minimum cross-entropy reconstruction of PET images using prior anatomical information", Physics in Medicine and Biologv, vol. 41, no. 11, pp. 2497-2517, 1996

[6] B. F. Hutton, A. Olsson, S. Som, K. Erlandsson, and M. Braun, "Reducing the influence of spatial resolution to improve quantitative accuracy in emission tomography: A comparison of potential strategies", Nuclear Instruments \& Methods in Physics Research Section aAccelerators Spectrometers Detectors and Associated Equipment, vol. 569, no. 2, pp. 462-466, 2006.

[7] S. Som, B. F. Hutton, and M. Braun, "Properties of minimum crossentropy reconstruction of emission tomography with anatomically based prior", IELE Transactions on Nuclear Science, vol. 45, no. 6, pp. 30143021, 1998.

[8] C. A. Johnson, Y. C. Yan, R. E. Carson, R. L. Martino, and M. E. Daube-Witherspoon, "A system for the 3D reconstruction of retractedsepta PET data using the EM algorithm", IEEE Transactions on Nuclear Science, vol. 42, no. 4, pp. 1223-1227, 1995.

[9] S. Jan, et al.,"GATE: a simulation toolkit for PET and SPECT", Physics in Medicine and Biology, vol. 49, no. 4, pp. 4543-4561, 2004 